\title{
Robustness of magnons near the quantum critical point in the heavy-fermion superconductor $\mathrm{CeCu}_{2} \mathrm{Si}_{2}$
}

\author{
Zita Huesges, ${ }^{1,2, *}$ Karin Schmalzl, ${ }^{3}$ Christoph Geibel, ${ }^{1}$ Manuel Brando, ${ }^{1}$ Frank Steglich, ${ }^{1}$ and Oliver Stockert ${ }^{1}$ \\ ${ }^{1}$ Max Planck Institute for Chemical Physics of Solids, 01187 Dresden, Germany \\ ${ }^{2}$ Helmholtz-Zentrum Berlin für Materialien und Energie GmbH, 14109 Berlin, Germany \\ ${ }^{3}$ Forschungszentrum Jülich GmbH, Jülich Centre for Neutron Science at ILL, 38000 Grenoble, France
}

(Received 20 March 2018; revised manuscript received 3 October 2018; published 16 October 2018)

\begin{abstract}
Paramagnons are supposed to provide the pairing glue for unconventional superconductors. For the heavyfermion superconductor $\mathrm{CeCu}_{2} \mathrm{Si}_{2}$, there is indeed good evidence from inelastic neutron scattering (INS) that spin fluctuations drive the superconductivity. Here, we present the INS measurement of the inelastic response of the antiferromagnetic parent compound, 'A-type' $\mathrm{CeCu}_{2} \mathrm{Si}_{2}$, to probe the relation to the excitations of the superconducting ('S-type') sample. We find that the dispersion is very similar in the antiferromagnetic state and in the normal state of the superconducting sample. Pronounced differences to the response in the superconducting state exist at low energies around the zone center. These findings are in line with observations of other unconventional superconductors.
\end{abstract}

DOI: 10.1103/PhysRevB.98.134425

Different classes of unconventional superconductorshigh- $T_{\mathrm{c}}$ cuprates, pnictides, and heavy-fermion superconductors-share a common feature in their phase diagrams, i.e., the appearance of a superconducting dome in the proximity of an antiferromagnetic quantum critical point (QCP) [1-5]. Major differences exist in other aspects of the phase diagrams, and the interactions leading to magnetic order are principally different. Nevertheless, it is assumed that for all these classes the magnetic excitations, associated with the ordered state, provide the pairing glue for superconductivity [6-8]. However, it is not a priori clear how the paramagnons in the superconducting compounds are related to the magnons of the antiferromagnetic phase, and microscopic measurements of the excitations are needed to shed light on this question.

For both cuprates and pnictides, advances in resonant inelastic x-ray scattering (RIXS) experiments have improved the knowledge of the magnetic excitations, in combination with results from inelastic neutron scattering (INS). It was shown that there are indeed remarkable similarities in the magnetic excitations of the antiferromagnetic and the superconducting phase. For the hole-doped cuprates, RIXS studies [913] revealed that paramagnons exist in the superconducting state even for overdoped samples, with similar intensity and dispersion relation to the magnons of the antiferromagnetic compound. An exception is the response near the magnetic zone center, where a sharp resonance is observed in the superconducting state. The width of the dispersive excitations increases in the doped samples due to the introduction of charge carriers. For electron-doped cuprates [14,15], intense paramagnons were also observed in RIXS, however their dispersion is shifted to higher energies.

*zita.huesges@helmholtz-berlin.de
Both RIXS and INS studies find paramagnons in the superconducting state of electron-doped Fe pnictides [16,17], which are softened but of similar overall intensity as the magnons in the parent compound. For hole-doped pnictides, paramagnons with a dispersion very similar to that of the magnons have been observed in INS $[16,18,19]$. As for the cuprates, significant differences appear close to the magnetic zone center.

For heavy-fermion compounds, spin excitation energies are about two orders of magnitude smaller than for cuprates and pnictides, in agreement with the difference in characteristic temperatures, and can thus be conveniently measured by INS. Among the heavy-fermion superconductors close to a magnetic QCP, dispersive excitations have been reported for $\mathrm{CePt}_{3} \mathrm{Si}$ [20], $\mathrm{CeCoIn}_{5}$ [21,22], and $\mathrm{CeCu}_{2} \mathrm{Si}_{2}$ [23,24]. In $\mathrm{CePt}_{3} \mathrm{Si}$ [20], magnetism and superconductivity coexist at low temperatures and no difference was found for the magnetic excitations of the purely magnetic and the mixed magneticsuperconducting state. In $\mathrm{CeCoIn}_{5}[21,22]$ and $\mathrm{CeCu}_{2} \mathrm{Si}_{2}$ $[23,24]$, a sharp peak appears within the superconducting energy gap at $0.6 \mathrm{meV}$ and $0.2 \mathrm{meV}$, respectively. At higher energies dispersive paramagnons are observed in both compounds. For $\mathrm{CeCoIn}_{5}$, these resemble the excitations of the antiferromagnetic reference compound $\mathrm{CeRhIn}_{5}[22,25]$. For $\mathrm{CeCu}_{2} \mathrm{Si}_{2}$, the dispersion in the superconducting state has been compared to the normal state $\left(T>T_{\mathrm{c}}\right.$ and $\left.B>B_{\mathrm{c} 2}\right)$ : The response is very similar for energies larger than $0.3 \mathrm{meV}$. However, measurements in the magnetic reference compound have so far been missing.

Here we present a comparative INS study of the magnetic excitations of antiferromagnetic ('A-type') and superconducting ('S-type') $\mathrm{CeCu}_{2} \mathrm{Si}_{2}$. Both ground states can be realized in homogeneous, quasistoichiometric samples of very similar composition, because the magnetic properties of $\mathrm{CeCu}_{2} \mathrm{Si}_{2}$ are highly sensitive to slight variations in the $\mathrm{Cu}$-to-Si ratio: They induce a small $(<1 \%)$ site exchange of $\mathrm{Si}$ by $\mathrm{Cu}$, 
which acts similar to hydrostatic pressure and tunes samples from an antiferromagnetic to a superconducting state [26,27]. This provides excellent conditions for neutron scattering, as both the antiferromagnetic and the superconducting state are accessible at ambient pressure. Additionally, the substitution of Si by Ge can enhance magnetic order [28]. We present dispersion measurements along [110] and along [001] for an 'A-type' crystal, both in the ordered phase and for $T>T_{\mathrm{N}}$, and show that the magnetic excitations resemble those of S-type $\mathrm{CeCu}_{2} \mathrm{Si}_{2}$.

Neutron scattering was performed at the cold triple axis spectrometer IN12 at ILL, Grenoble, on a $3.0 \mathrm{~g}$ single crystal of $\mathrm{CeCu}_{2}\left(\mathrm{Si}_{0.98} \mathrm{Ge}_{0.02}\right)_{2}$. Details of the experiment as well as on sample characterization can be found in the Supplemental Material [32]. Both elastic neutron scattering and thermodynamic data revealed an antiferromagnetic phase below $T_{\mathrm{N}}=$ $0.85 \mathrm{~K}$. The ordering wave vector at the lowest measured temperature of $0.1 \mathrm{~K}$ was found to be $\tau=(0.2150 .215$ $0.52)$; it can be more easily measured in the second Brillouin zone at $Q_{\mathrm{AF}}=\left(\begin{array}{lll}0.215 & 0.215 & 1.48\end{array}\right)$, due to a much larger signal-to-background ratio. The value of $\tau$, particularly the $h$ component, is temperature dependent above $0.4 \mathrm{~K}$, reaching $h \approx 0.228$ around the Néel temperature. These observations are very similar to measurements of A-type $\mathrm{CeCu}_{2} \mathrm{Si}_{2}$ samples without Ge doping [30,31]. As outlined in the Supplemental Material [32], a minor fraction of the sample gradually turns superconducting below $0.4 \mathrm{~K}$. Therefore, data in the antiferromagnetic phase were collected at $0.4 \mathrm{~K}$ [32].

The inelastic response has been measured by constant- $Q$ scans at the ordering wave vector and by constant- $E$ scans for the study of the dispersion. The spectra measured at $Q_{\mathrm{AF}}$ at $0.4 \mathrm{~K}$ and $5 \mathrm{~K}$ are shown in Fig. 1(a). The total response is comprised of an incoherent elastic contribution (temperature-
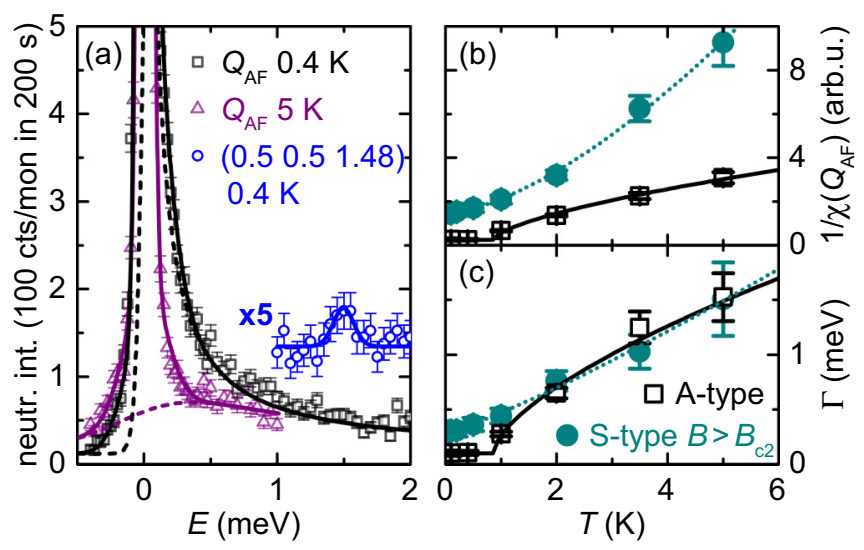

FIG. 1. (a) Spectra of $\mathrm{CeCu}_{2} \mathrm{Si}_{2}$ at $0.4 \mathrm{~K}$ (black) and $5 \mathrm{~K}$ (purple), measured at $Q_{\mathrm{AF}}=(0.2150 .2151 .48)$; solid black/purple lines show total fit functions, dashed the inelastic magnetic contribution, which was fitted with a Bose-weighted Lorentzian function. Shown in blue is a scan at the magnetic zone boundary $Q=(0.50 .51 .48)$ at $0.4 \mathrm{~K}$ with a Gaussian fit; these data have been magnified by a factor of 5. (b) Fitted inverse susceptibility $1 / \chi$ and (c) width $\Gamma$ (full width at half maximum, FWHM) of the inelastic magnetic signal at $Q_{\mathrm{AF}}$, for our A-type crystal and for the S-type crystal at $1.7 \mathrm{~T}$ (taken from Ref. [23]). $\chi\left(Q_{\mathrm{AF}}\right)$ is normalized to the intensity of the incoherent elastic line [29]. Lines are guides to the eye.
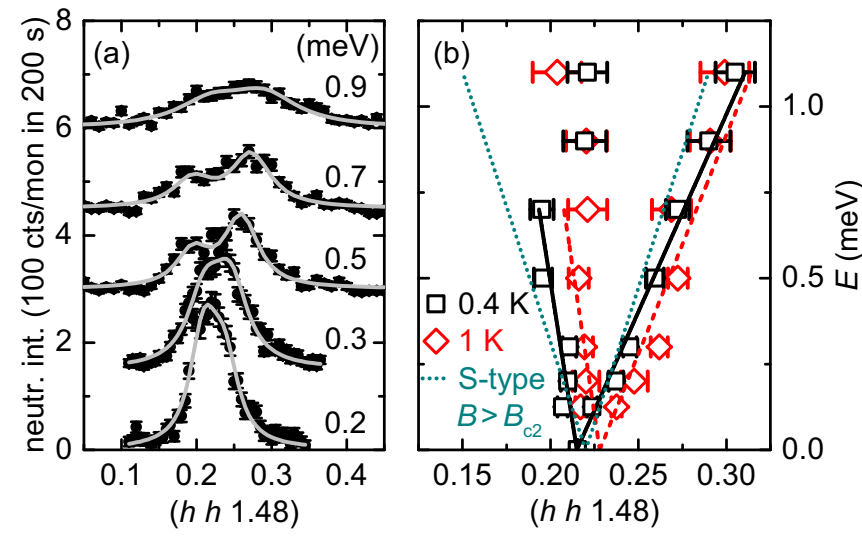

FIG. 2. Dispersion of the magnetic mode in A-type $\mathrm{CeCu}_{2} \mathrm{Si}_{2}$. (a) Scans along [110] at $0.4 \mathrm{~K}$ for several energy transfers, as well as Lorentzian fit functions (solid lines). Data are displayed after background subtraction and with an offset of 150 counts. (b) Fitted peak positions of the magnetic mode for $0.4 \mathrm{~K}$ and $1 \mathrm{~K}$. Linear fits for the dispersion relation are shown in solid or dashed lines, respectively (details see main text). The dotted line shows the dispersion of S-type $\mathrm{CeCu}_{2} \mathrm{Si}_{2}$ in the normal state $(B=1.7 \mathrm{~T}$, $T=60 \mathrm{mK})$ [23].

independent), a quasielastic magnetic response, and an elastic magnetic signal (present only below $T_{\mathrm{N}}$ ). The quasielastic component can be fitted with a Lorentzian function including the Bose temperature factor [32]. The fitted inverse susceptibility $1 / \chi\left(Q_{\mathrm{AF}}\right)$ and width $\Gamma$ (FWHM, inverse lifetime) for all measured temperatures are plotted in Figs. 1(b) and 1(c), respectively. Below $T_{\mathrm{N}}$, it is difficult to separate the dynamic response from the static magnetic signal, so that absolute values for $\chi$ and $\Gamma$ are not very accurate. Still, it is clear that both the susceptibility and the lifetime diverge in the antiferromagnetic state.

In Fig. 2(a), constant- $E$ scans along [110] around $Q_{\mathrm{AF}}$ are displayed, together with Lorentzian fit curves. Two peaks of identical width are fitted to each curve to account for the dispersive splitting. We chose a simple Lorentzian fit approach, because the data are not sufficient for a full, modelbased fit of the dispersion relation. The main reasons for this insufficiency are the strong damping at higher energy transfers and the incommensurability of the magnetic order. For incommensurate order, multiple overlapping branches may exist, and the resulting intensity distribution is not necessarily symmetric with respect to the zone center. This is also seen, for example, in the dispersion relation of CeRhIn 5 along [001] [25]. For $\mathrm{CeCu}_{2} \mathrm{Si}_{2}$, we see a strong asymmetry of the intensity of the V-shaped dispersion, which increases towards higher energy transfer. The fitted peak positions are displayed in Fig. 2(b). At $0.9 \mathrm{meV}$ and $1.1 \mathrm{meV}$, the position of the weaker branch cannot be extracted reliably. The fitted peak positions suggest linear spin-wave dispersions with spinwave velocities of $5.3 \pm 0.2 \mathrm{meV} \AA$ (upper branch, fitted up to $1.1 \mathrm{meV}$ ) and $15 \pm 3 \mathrm{meV} \AA$ [lower branch, fitted up to $0.7 \mathrm{meV}$, see Fig. 2(b)]. However, we note that in case of overlapping dispersion branches it is difficult to extract values for the velocities based on a single linear fit. Our data give no indication of an anisotropy gap in the ordered state (within 


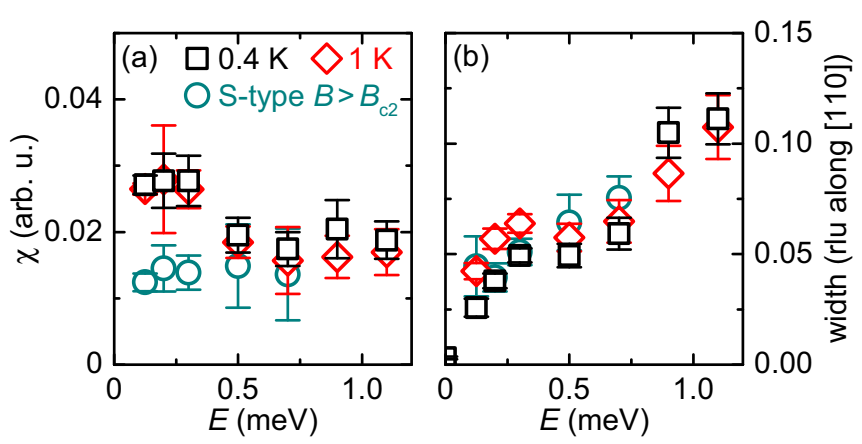

FIG. 3. (a) Susceptibility associated with the constant- $E$ scans, obtained from the integrated intensity over both branches of the dispersion and further corrected for the Bose temperature factor. The data for the S-type crystal are taken in the normal state at $1.7 \mathrm{~T}$ and $60 \mathrm{mK}$. The same normalization to the incoherent elastic line has been done as in Fig. 1 [29]. (b) Width (FWHM) of the constant- $E$ scans along [110].

the energy resolution of $65 \mu \mathrm{eV} \mathrm{FWHM),} \mathrm{as} \mathrm{it} \mathrm{was} \mathrm{observed}$ in the dispersion of $\mathrm{CePd}_{2} \mathrm{Si}_{2}$ [33], $\mathrm{CeIn}_{3}$ [34], $\mathrm{CeRhIn} 5$ [25], and $\mathrm{CeCu}_{2} \mathrm{Ge}_{2}$ [35]. A more detailed discussion of our fits, particularly resolution effects, is presented in the Supplemental Material [32].

In Fig. 3, susceptibility and width of the fitted magnetic signal are displayed. While the susceptibility slowly decreases with energy transfer, the width strongly increases. Even at small energy transfers, the excitations are strongly damped, the inelastic correlation length being roughly $26 \AA$ [36]. At $\Delta E \approx 1 \mathrm{meV}$, the inelastic correlation length drops to values corresponding to nearest-neighbor interaction. The origin of the increased damping might be enhanced Kondo scattering, since the energy scale is similar to the local spin-fluctuation temperature of $\mathrm{CeCu}_{2} \mathrm{Si}_{2}$, which was observed in powder neutron spectroscopy [37]. Furthermore, overlapping dispersion branches might influence the fitted width. Due to the strong broadening of the signal, it is difficult to follow the dispersion further towards the magnetic zone boundary. In a constant- $Q$ scan at (0.5 0.5 1.48) [cf. Fig. 1(a)], a weak maximum can be seen at $1.5 \mathrm{meV}$, which seems rather sharp considering the damping observed in the constant- $E$ scans. Therefore, it is not clear whether it can be identified with the bending of the dispersion.

In Figs. 2 and 3, we also include fit parameters for data measured at $1 \mathrm{~K}$. Although this is above $T_{\mathrm{N}}$, only minor changes are observed to the magnetic excitations. The most noticeable difference is the shift of the center of the dispersion, which is in agreement with the shift of the ordering wave vector up to $T_{\mathrm{N}}=0.85 \mathrm{~K}$. A slight increase is observed in the width of the low-energy excitations, but it does not reflect the divergence of the static correlation length. Similarly, the divergence of the susceptibility at $E \rightarrow 0$ is not reflected in the dispersive excitations.

We now turn to the dispersion along the [001] direction, which has been measured at energy transfers between 0.125 and $0.7 \mathrm{meV}$. For a better comparison with the dispersion along [110], all data have been transformed from reciprocal lattice units (rlu) to absolute momentum transfer $\left(\AA^{-1}\right)$ [38] and shifted such that the center of the dispersion $(0.215$ rlu
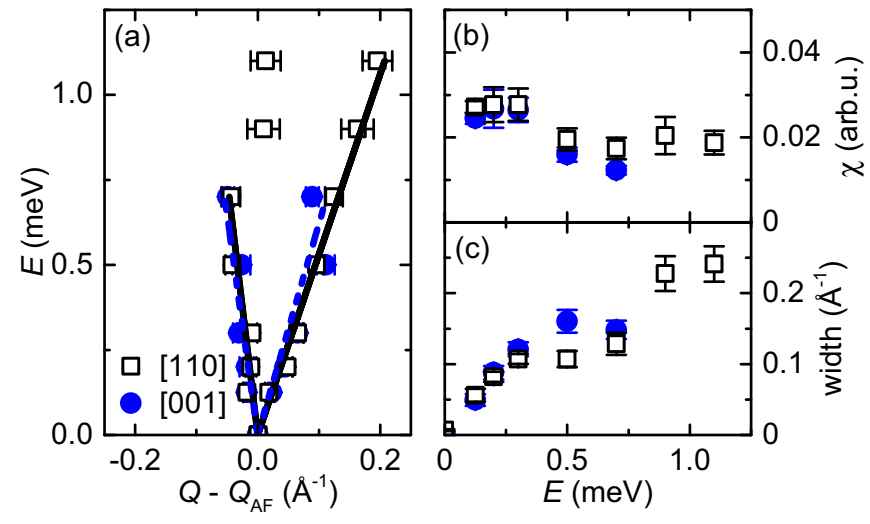

FIG. 4. (a) Fitted peak positions, as well as linear dispersion relations, for constant- $E$ scans along [110] and [001] at $0.4 \mathrm{~K}$. The data along [110] are the same as in Fig. 2, but for better comparison the axis has been transformed from rlu to $\AA^{-1}$ [38] and the center of the dispersion set to zero. (b) and (c) Comparison of susceptibility and width (FWHM) of constant- $E$ scans along [001] with data along [110] already shown in Fig. 3.

or $1.48 \mathrm{rlu}$ ) is at zero. Resolution effects are accounted for by a convolution with an appropriate Gaussian function for the [110] and the [001] direction, respectively. The fitted peak positions are displayed in Fig. 4(a). Within error bars, the dispersion relations along both directions are the same. We note that the [001] direction shows the same asymmetry of the spin-wave velocities for both branches as the [110] direction, while it does not exhibit any asymmetry in the intensity of the branches. As for the [110] direction, data at $1 \mathrm{~K}$ (not shown) closely resemble those at $0.4 \mathrm{~K}$. From Figs. 4(b) and 4(c) it is furthermore evident that the susceptibility and the width of the constant- $E$ scans are very similar for the two directions.

The dispersion analysis of A-type $\mathrm{CeCu}_{2} \mathrm{Si}_{2}$ has shown that damped magnons exist both in the antiferromagnetic and in the paramagnetic phase just above $T_{\mathrm{N}}$. In contrast to many other antiferromagnetic heavy-fermion systems [25,3335], the dispersion is gapless; this, together with the quite small ordered moment of $0.1 \mu_{\mathrm{B}} / \mathrm{Ce}$ [30], reflects that Atype $\mathrm{CeCu}_{2} \mathrm{Si}_{2}$ is located very close to the quantum critical point - and thus also to the superconducting phase. We could also demonstrate that the dispersion relation and the spectral weight contributions are almost identical for the [110] and the [001] direction, indicating the truly three-dimensional nature of the magnetic interactions. Similarly, three-dimensional interactions have been seen in $\mathrm{CeCoIn}_{5}$ (also tetragonal) in the superconducting state [22]. This has served as an argument against an exciton scenario in $\mathrm{CeCoIn}_{5}$ [39], since the question whether the magnetic interactions are (quasi-)2D or $3 \mathrm{D}$ is important for the superconducting pairing mechanism. Analogously, our observations question that the exciton scenario is appropriate for $\mathrm{CeCu}_{2} \mathrm{Si}_{2}$.

To compare the inelastic response of A-type $\mathrm{CeCu}_{2} \mathrm{Si}_{2}$ to that of S-type $\mathrm{CeCu}_{2} \mathrm{Si}_{2}$ [23,24], we included data of the S-type crystal in Figs. 1, 2, and 3. A direct comparison of the data is also shown in the contour plots in Figs. 5(a) and 5(b). The data of S-type $\mathrm{CeCu}_{2} \mathrm{Si}_{2}$ are measured in the normal state at $B>B_{\mathrm{c} 2}$; in Ref. [23], it was reported that the normal state 


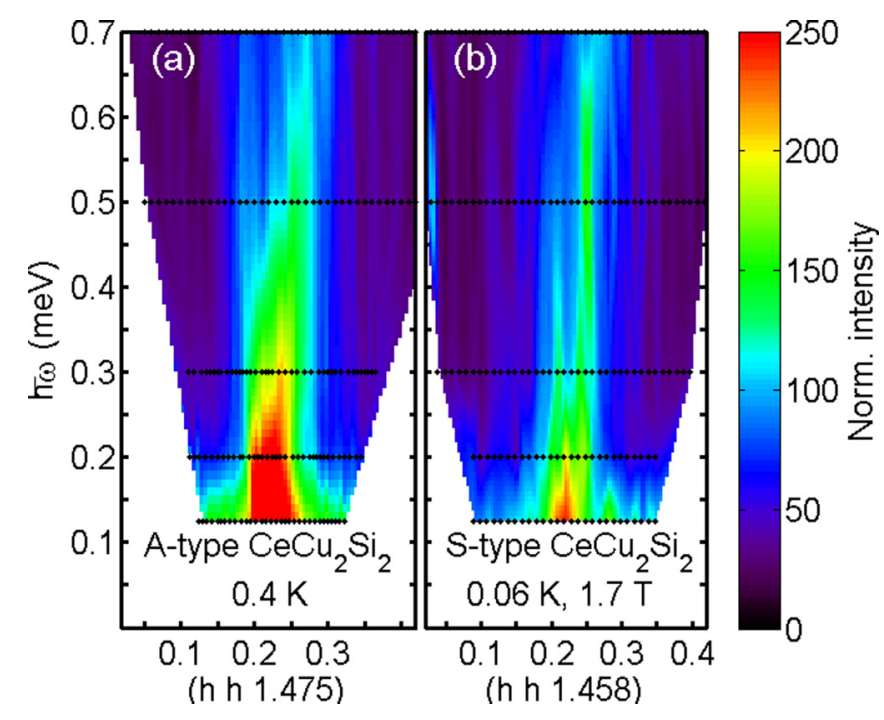

FIG. 5. Contour plots of the dispersion of (a) A-type $\mathrm{CeCu}_{2} \mathrm{Si}_{2}$ and (b) S-type $\mathrm{CeCu}_{2} \mathrm{Si}_{2}$, the latter measured in the normal state at $B>B_{\mathrm{c} 2}$ [23]. The black dots show the actual measurement positions. The color scale denotes neutron counts, normalized to the incoherent line [29]. A corresponding image of the S-type in the superconducting state can be found in the publication by Stockert et al. [24].

at $T>T_{\mathrm{C}}$ has very similar excitations. Figure 2 shows that the dispersion relation of S-type $\mathrm{CeCu}_{2} \mathrm{Si}_{2}$ in the normal state resembles that of A-type $\mathrm{CeCu}_{2} \mathrm{Si}_{2}$. The fit for the S-type sample was done under the assumption that both branches have the same spin-wave velocity [23]; a free fit also results in different velocities for both branches, however not quite as different as for the A-type sample. The pronounced asymmetry of the intensity of the two dispersion branches is also seen for $\mathrm{S}$-type $\mathrm{CeCu}_{2} \mathrm{Si}_{2}$. The inelastic response of A-type and $\mathrm{S}$-type $\mathrm{CeCu}_{2} \mathrm{Si}_{2}$ differs concerning the susceptibility, which is larger for the A-type crystal by about a factor of 2.5 already above $T_{\mathrm{N}}$ [cf. Fig. 1(b)]. Figure 3(a) shows that the increase in intensity mainly happens at low energy transfers. This reflects the increase of the Kondo effect in the S-type sample, which prevents the divergence of the susceptibility at $Q_{\mathrm{AF}}$. Still, the increase in Kondo scattering does not lead to an increase of the damping: Both the lifetime [Fig. 1(c)] and the momentum transfer width [Fig. 3(c)] have roughly the same magnitude for both compounds.

Thus, the excitations of the A-type crystal (AF or PM) and the S-type crystal (normal state) are very similar except for the reduction of intensity at low energy transfers. This suggests that the magnetic exchange interaction is nearly identical in both compounds, in accordance with their close proximity in the phase diagram. Stockert et al. [24] have discussed the differences between the normal and the superconducting state of S-type $\mathrm{CeCu}_{2} \mathrm{Si}_{2}$ : A spin gap of $\approx 0.2 \mathrm{meV}$ opens below $T_{\mathrm{c}}$, accompanied by a strong increase of spectral intensity just above the gap. It is demonstrated that there is an exchange energy saving of roughly $5 \mu \mathrm{eV}$ per Ce connected to the spectral weight shift, which can account for the superconducting condensation energy. With the data of the A-type crystal at hand, we can now conclude that a similar spectral weight shift and a comparable exchange energy saving exists between the antiferromagnetic and the superconducting state, i.e., when moving along the pressure axis in the phase diagram rather than the temperature or field axis. This analogy is possible only because the antiferromagnetic state, as the normal state of the S-type sample, has a gapless dispersion.

Our comparison of the superconductor and its antiferromagnetic reference compound yields the same general trend for $\mathrm{CeCu}_{2} \mathrm{Si}_{2}$ as for cuprates [9-15], pnictides [16-19], and $\mathrm{CeCoIn}_{5}$ [22,25]: The significant differences in the inelastic response appear around the zone center at low energies, while the dispersive excitations at higher energies are very similar.

It is interesting to extend the comparison of $\mathrm{CeCu}_{2} \mathrm{Si}_{2}$ to other superconductors with respect to the nature of the "resonance." In Ref. [24], it was assumed that the peak observed in INS lies just above the charge gap, leading to a principally different situation than in the cuprates or $\mathrm{CeCoIn}_{5}$. However, a multitude of new measurements indicate that $\mathrm{CeCu}_{2} \mathrm{Si}_{2}$ is a two-band superconductor [40-44], with one gap being significantly larger than the other $\left(\Delta_{1} \approx 4-5 \Delta_{2}\right.$ [43]). $\Delta_{1} \approx 5 k_{B} T_{\mathrm{c}}$ is in line with $\mathrm{Cu}-\mathrm{NQR}$ results [45] and scanning tunneling spectroscopy [41]. This leaves the spin excitation just above the spin gap of $3.9 k_{B} T_{\mathrm{c}}$ as an in-gap state. Thus, the situation might be analogous to that of $\mathrm{CeCoIn}_{5}$.

As for cuprates it was initially assumed for $\mathrm{CeCoIn}_{5}$ that the resonance is an excitonic excitation within the charge gap. However, recent INS experiments on $\mathrm{Yb}$ substituted $\mathrm{CeCoIn}_{5}$ suggest that the origin of the peak is a magnon rather than an exciton [22]. The main arguments against the exciton scenario are the lack of a downward dispersion towards lower energies and the 3D nature of the interactions [22,39], which both apply to $\mathrm{CeCu}_{2} \mathrm{Si}_{2}$ as well. Within the magnon scenario, the appearance of the sharp peak is explained by a strong decrease of the damping in the superconducting state. While the exciton scenario implies a sign changing superconducting order parameter, no such implication exists in the magnon scenario.

In conclusion, we have reported on the INS measurement of the dispersion of antiferromagnetic $\mathrm{CeCu}_{2} \mathrm{Si}_{2}$. We show that the inelastic response closely resembles that of superconducting $\mathrm{CeCu}_{2} \mathrm{Si}_{2}$, except near the zone center at low energies. These findings are similar to observations in cuprates, pnictides, and $\mathrm{CeCoIn}_{5}$, reinforcing the idea of a common, magnetic driving force for superconductivity.

We thank Silvia Seiro for her advice in the single crystal synthesis, Bernhard Keimer, Dmytro Inosov, and Michael Loewenhaupt for productive discussions, and Tobias Weber for support with resolution calculations using Takin. We acknowledge financial support by the DFG research unit FOR 960 'Quantum Phase Transitions.'
[1] M. R. Norman and C. Pépin, Rep. Prog. Phys. 66, 1547 (2003).
[2] K. M. Shen and J. S. Davis, Mater. Today 11, 14 (2008). 
[3] P. J. Hirschfeld, M. M. Korshunov, and I. I. Mazin, Rep. Prog. Phys. 74, 124508 (2011).

[4] G. R. Stewart, Rev. Mod. Phys. 83, 1589 (2011).

[5] C. Pfleiderer, Rev. Mod. Phys. 81, 1551 (2009).

[6] D. J. Scalapino, Rev. Mod. Phys. 84, 1383 (2012).

[7] P. Monthoux, D. Pines, and G. G. Lonzarich, Nature (London) 450, 1177 (2007).

[8] T. Dahm, V. Hinkov, S. V. Borisenko, A. A. Kordyuk, V. B. Zabolotnyy, J. Fink, B. Buchner, D. J. Scalapino, W. Hanke, and B. Keimer, Nat. Phys. 5, 217 (2009).

[9] M. Le Tacon, G. Ghiringhelli, J. Chaloupka, M. M. Sala, V. Hinkov, M. W. Haverkort, M. Minola, M. Bakr, K. J. Zhou, S. Blanco-Canosa, C. Monney, Y. T. Song, G. L. Sun, C. T. Lin, G. M. De Luca, M. Salluzzo, G. Khaliullin, T. Schmitt, L. Braicovich, and B. Keimer, Nat. Phys. 7, 725 (2011).

[10] M. Le Tacon, M. Minola, D. C. Peets, M. Moretti Sala, S. Blanco-Canosa, V. Hinkov, R. Liang, D. A. Bonn, W. N. Hardy, C. T. Lin, T. Schmitt, L. Braicovich, G. Ghiringhelli, and B. Keimer, Phys. Rev. B 88, 020501 (2013).

[11] M. P. M. Dean, A. J. A. James, R. S. Springell, X. Liu, C. Monney, K. J. Zhou, R. M. Konik, J. S. Wen, Z. J. Xu, G. D. Gu, V. N. Strocov, T. Schmitt, and J. P. Hill, Phys. Rev. Lett. 110, 147001 (2013).

[12] M. P. M. Dean, G. Dellea, R. S. Springell, F. Yakhou-Harris, K. Kummer, N. B. Brookes, X. Liu, Y.-J. Sun, J. Strle, T. Schmitt, L. Braicovich, G. Ghiringhelli, I. Božović, and J. P. Hill, Nat. Mater. 12, 1019 (2013).

[13] M. Minola, G. Dellea, H. Gretarsson, Y. Y. Peng, Y. Lu, J. Porras, T. Loew, F. Yakhou, N. B. Brookes, Y. B. Huang, J. Pelliciari, T. Schmitt, G. Ghiringhelli, B. Keimer, L. Braicovich, and M. Le Tacon, Phys. Rev. Lett. 114, 217003 (2015).

[14] K. Ishii, M. Fujita, T. Sasaki, M. Minola, G. Dellea, C. Mazzoli, K. Kummer, G. Ghiringhelli, L. Braicovich, T. Tohyama, K. Tsutsumi, K. Sato, R. Kajimoto, K. Ikeuchi, K. Yamada, M. Yoshida, M. Kurooka, and J. Mizuki, Nat. Commun. 5, 3714 (2014).

[15] W. S. Lee, J. J. Lee, E. A. Nowadnick, S. Gerber, W. Tabis, S. W. Huang, V. N. Strocov, E. M. Motoyama, G. Yu, B. Moritz, H. Y. Huang, R. P. Wang, Y. B. Huang, W. B. Wu, C. T. Chen, D. J. Huang, M. Greven, T. Schmitt, Z. X. Shen, and T. P. Devereaux, Nat. Phys. 10, 883 (2014).

[16] M. Wang, C. Zhang, X. Lu, G. Tan, H. Luo, Y. Song, M. Wang, X. Zhang, E. A. Goremychkin, T. G. Perring, T. A. Maier, Z. Yin, K. Haule, G. Kotliar, and P. Dai, Nat. Commun. 4, 2874 (2013).

[17] K.-J. Zhou, Y.-B. Huang, C. Monney, X. Dai, V. N. Strocov, N.-L. Wang, Z.-G. Chen, C. Zhang, P. Dai, L. Patthey, J. van den Brink, H. Ding, and T. Schmitt, Nat. Commun. 4, 1470 (2013).

[18] M. Liu, L. W. Harriger, H. Luo, M. Wang, R. A. Ewings, T. Guidi, H. Park, K. Haule, G. Kotliar, S. M. Hayden, and P. Dai, Nat. Phys. 8, 376 (2012).

[19] H. Luo, X. Lu, R. Zhang, M. Wang, E. A. Goremychkin, D. T. Adroja, S. Danilkin, G. Deng, Z. Yamani, and P. Dai, Phys. Rev. B 88, 144516 (2013).

[20] B. Fåk, S. Raymond, D. Braithwaite, G. Lapertot, and J.-M. Mignot, Phys. Rev. B 78, 184518 (2008).

[21] C. Stock, C. Broholm, J. Hudis, H. J. Kang, and C. Petrovic, Phys. Rev. Lett. 100, 087001 (2008).

[22] Y. Song, J. Van Dyke, I. K. Lum, B. D. White, S. Jang, D. Yazici, L. Shu, A. Schneidewind, P. Čermák, Y. Qiu,
M. B. Maple, D. K. Morr, and P. Dai, Nat. Commun. 7, 12774 (2016).

[23] J. Arndt, O. Stockert, K. Schmalzl, E. Faulhaber, H. S. Jeevan, C. Geibel, W. Schmidt, M. Loewenhaupt, and F. Steglich, Phys. Rev. Lett. 106, 246401 (2011).

[24] O. Stockert, J. Arndt, E. Faulhaber, C. Geibel, H. S. Jeevan, S. Kirchner, M. Loewenhaupt, K. Schmalzl, W. Schmidt, Q. Si, and F. Steglich, Nat. Phys. 7, 119 (2011).

[25] P. Das, S.-Z. Lin, N. J. Ghimire, K. Huang, F. Ronning, E. D. Bauer, J. D. Thompson, C. D. Batista, G. Ehlers, and M. Janoschek, Phys. Rev. Lett. 113, 246403 (2014).

[26] F. Steglich, P. Gegenwart, C. Geibel, R. Helfrich, P. Hellmann, M. Lang, A. Link, R. Modler, G. Sparn, N. Büttgen, and A. Loidl, Physica B: Condensed Matter 223-224, 1 (1996).

[27] S. Seiro, M. Deppe, H. Jeevan, U. Burkhardt, and C. Geibel, Phys. Status Solidi (b) 247, 614 (2010).

[28] H. Q. Yuan, F. M. Grosche, M. Deppe, C. Geibel, G. Sparn, and F. Steglich, Science 302, 2104 (2003).

[29] The incoherent elastic line was used for intensity comparison between the A- and S-type sample, since it is mainly caused by scattering from the $\mathrm{Cu}$ nuclei and not very sensitive to the content of $\mathrm{Ce}, \mathrm{Si}$, and $\mathrm{Ge}$.

[30] O. Stockert, E. Faulhaber, G. Zwicknagl, N. Stüßer, H. S. Jeevan, M. Deppe, R. Borth, R. Küchler, M. Loewenhaupt, C. Geibel, and F. Steglich, Phys. Rev. Lett. 92, 136401 (2004).

[31] O. Stockert, E. Faulhaber, K. Schmalzl, W. Schmidt, H. S. Jeevan, M. Deppe, C. Geibel, T. Cichorek, T. Nakanishi, M. Loewenhaupt, and F. Steglich, J. Phys.: Conf. Ser. 51, 211 (2006).

[32] See Supplemental Material at http://link.aps.org/supplemental/ 10.1103/PhysRevB.98.134425 for more details on the experiment, data fitting, and the effects of instrument resolution.

[33] N. van Dijk, B. Fåk, T. Charvolin, P. Lejay, J. Mignot, and B. Hennion, Physica B: Condensed Matter 241-243, 808 (1997).

[34] W. Knafo, S. Raymond, B. Fåk, G. Lapertot, P. C. Canfield, and J. Flouquet, J. Phys.: Condens. Matter 15, 3741 (2003).

[35] P. Geselbracht, E. Faulhaber, M. Rotter, K. Schmalzl, D. Quintero-Castro, O. Stockert, M. Loewenhaupt, and A. Schneidewind, Phys. Proc. 75, 83 (2015).

[36] We define the inelastic correlation length as the inverse of the half width at half maximum, extracted from the Lorentzian fits to the constant- $Q$ scans.

[37] S. Horn, E. Holland-Moritz, M. Loewenhaupt, F. Steglich, H. Scheuer, A. Benoit, and J. Flouquet, Phys. Rev. B 23, 3171 (1981).

[38] For the [110] direction, $1 \mathrm{rlu}=2 \pi \sqrt{2} / a=2.17 \AA^{-1}$, and for the [001] direction, $1 \mathrm{rlu}=2 \pi / c=0.63 \AA^{-1}$.

[39] A. V. Chubukov and L. P. Gor'kov, Phys. Rev. Lett. 101, 147004 (2008).

[40] S. Kittaka, Y. Aoki, Y. Shimura, T. Sakakibara, S. Seiro, C. Geibel, F. Steglich, H. Ikeda, and K. Machida, Phys. Rev. Lett. 112, 067002 (2014).

[41] M. Enayat, Z. Sun, A. Maldonado, H. Suderow, S. Seiro, C. Geibel, S. Wirth, F. Steglich, and P. Wahl, Phys. Rev. B 93, 045123 (2016).

[42] S. Kittaka, Y. Aoki, Y. Shimura, T. Sakakibara, S. Seiro, C. Geibel, F. Steglich, Y. Tsutsumi, H. Ikeda, and K. Machida, Phys. Rev. B 94, 054514 (2016). 
[43] G. Pang, M. Smidman, J. Zhang, L. Jiao, Z. Weng, E. M. Nica, Y. Chen, W. Jiang, Y. Zhang, W. Xie, H. S. Jeevan, H. Lee, P. Gegenwart, F. Steglich, Q. Si, and H. Yuan, Proc. Natl. Acad. Sci. USA 115, 5343 (2018).

[44] T. Yamashita, T. Takenaka, Y. Tokiwa, J. A. Wilcox, Y. Mizukami, D. Terazawa, Y. Kasahara, S. Kittaka, T. Sakak- ibara, M. Konczykowski, S. Seiro, H. S. Jeevan, C. Geibel, C. Putzke, T. Onishi, H. Ikeda, A. Carrington, T. Shibauchi, and Y. Matsuda, Sci. Adv. 3, e1601667 (2017).

[45] K. Fujiwara, Y. Hata, K. Kobayashi, K. Miyoshi, J. Takeuchi, Y. Shimaoka, H. Kotegawa, T. C. Kobayashi, C. Geibel, and F. Steglich, J. Phys. Soc. Jpn. 77, 123711 (2008). 